\title{
Research Paper \\ Investigating of Factors Affecting the Fertility Pattern of Arak City in 2018 With the Structural Equation Modeling
}

\author{
Fatemeh Amiri $^{1} \odot$, Rasoul Najafi $^{1} \mathrm{C}$
}

1. Department of Biostatistics, School of Medicine, Arak University of Medical Sciences, Arak, Iran

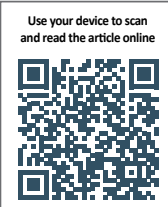

Citation: Amiri F, Najafi R. [Investigating of Factors Affecting the Fertility Pattern of Arak City in 2018 With the Structural Equation Modeling (Persian)]. Journal of Arak University of Medical Sciences(JAMS). 2020; 23(3):360-373. https://doi. org/10.32598/JAMS.23.3.5531.3

https://doi.org/10.32598/JAMS.23.3.5531.3

Key words:

Fertility, Structural equation modeling, Contraception

\section{A B S TRACT}

Article Info:

Received: 25 Feb 2020

Accepted: 17 May 2020

Available Online: 01 Aug 2020

\section{Extended Abstract}

\section{Introduction}

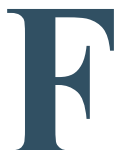

ertility pattern is the most important phenomenon determining population fluctuations [13] which is defined based on the birth interval between children [4]. The total fertility rate for each woman is 2.5 children $[5,6]$. Infertility is a central issue for the gestational ages of couples around the world. A systematic analysis of infertility in 190 countries showed that in 2010, women aged 20-44 suffered 1.9 percent of primary infertility and 10.5 percent of secondary infertility [7]. In Iran, the prevalence of infertility was reported to be $12-21.9 \%$ [8]. Demographic factors such as education, age at marriage and lifestyle factors affect fertility [9].

\section{* Corresponding Author:}

Rasoul Najafi, MSc.

Address: Department of Biostatistics, School of Medicine, Arak University of Medical Sciences, Arak, Iran.

Tel: +98 (910) 8010934

E-mail: rasolnajafi@yahoo.com 


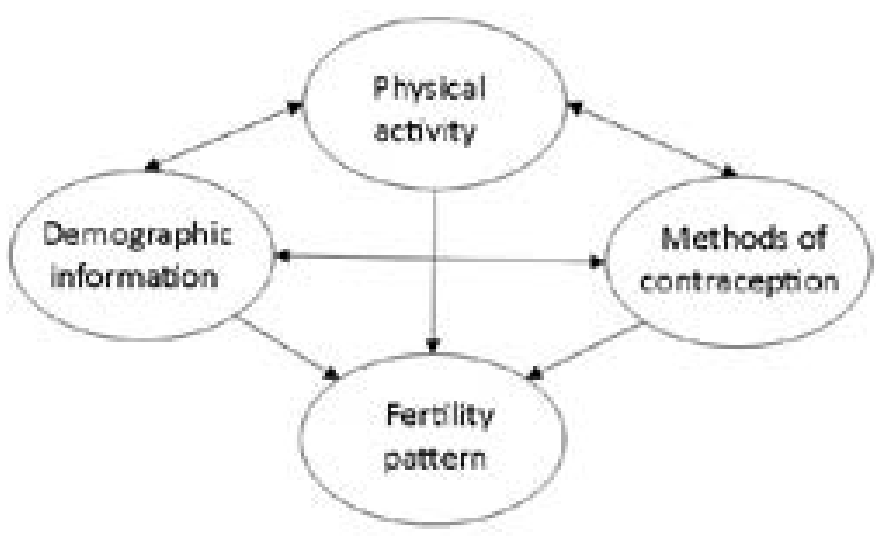

Figure 1. Hypothetical conceptual model of relationships between variables

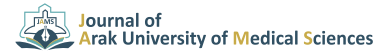

One way to determine the factors affecting the fertility pattern is to use Structural Equation Modeling (SEM), which allows the researcher to simultaneously examine the expression relationships of variables [10] (Table 1). The present study was conducted to investigate the factors affecting the fertility pattern of women in Arak.

\section{Materials and Methods}

The present study was a cross-sectional study in which the statistical population consisted of all married women of childbearing age who took care of patients. These women, who referred to three hospitals of "Taleghani Educational-
Medical Center", gynecological surgery ward of "Valiasr Educational-Medical Center" and pediatric ward of "Amirkabir Educational-Medical Center" in Arak in 2017, were randomly selected. A total of 384 married women aged 15-49 years participated in this study.

The first part of the checklist consisted of demographic information (age, education, place of residence, occupation, and income level) and the second part consisted of questions including methods of contraception, infertility history, infertility treatment, and physical activity. Structural models determined which of the independent variables affected which of the dependent variables or which variables

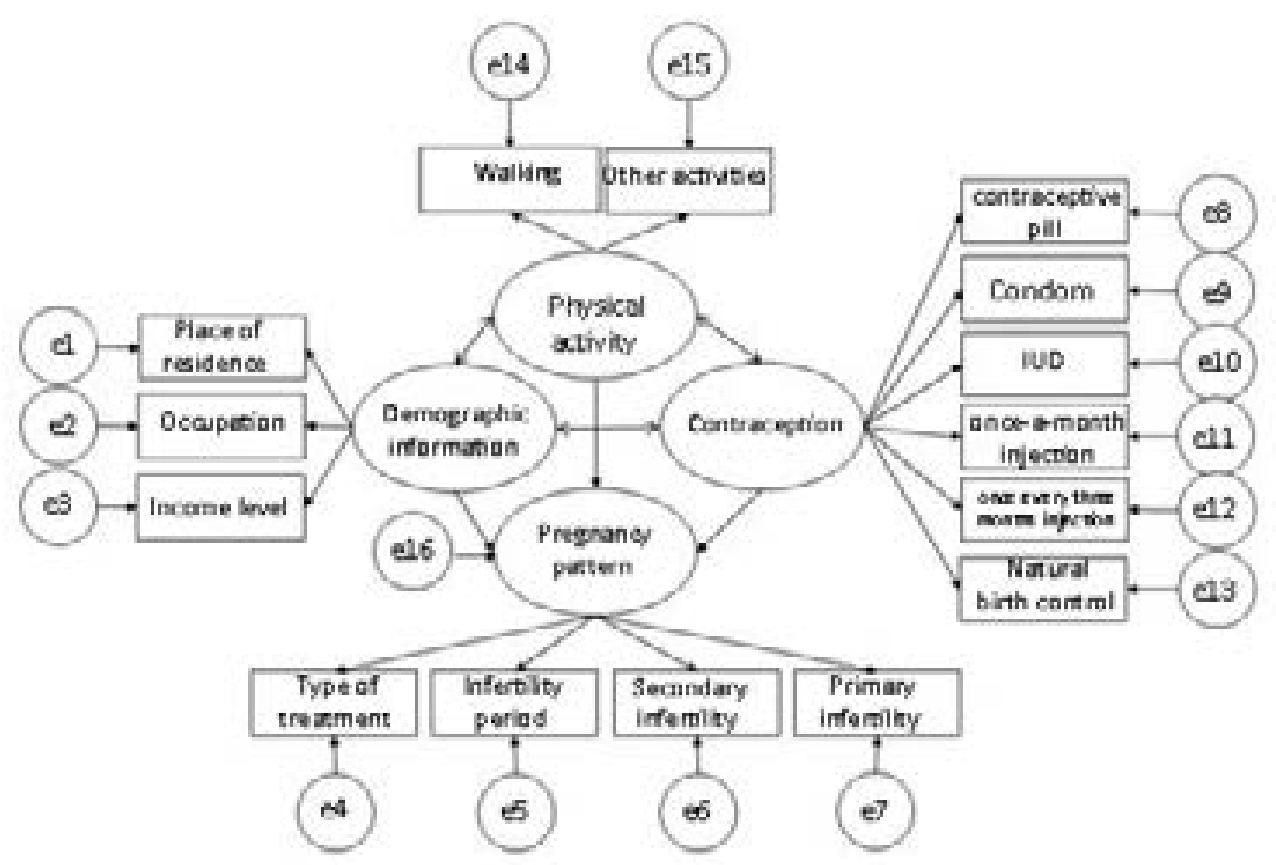

Figure 2. Fertility pattern, methods of contraception, physical activity and demographic information 
Table 1. Fitness indicators related to the proposed model

\begin{tabular}{|c|c|c|c|}
\hline Fitness Index & Developed Model & Acceptable Domain & Ideal Domain \\
\hline$X^{2} / \mathrm{df}$ & 2.32 & $X^{2} / \mathrm{df} \leq 5$ & $X^{2} / \mathrm{df} \leq 2$ \\
\hline GFI & 0.96 & $0.9 \leq \mathrm{GF} \mid<0.95$ & $0.95 \leq \mathrm{GFI}<1$ \\
\hline CFI & 0.97 & $0.9 \leq \mathrm{CF} \mid<0.95$ & $0.95 \leq \mathrm{CF}<1$ \\
\hline PCFI & 0.96 & $0.9 \leq \mathrm{PCF} \mid<0.95$ & $0.95 \leq \mathrm{PCF}<1$ \\
\hline AGFI & 0.95 & $0.9 \leq \mathrm{AGFI}<0.95$ & $0.95 \leq \mathrm{AGFI}<1$ \\
\hline RMSEA & 0.06 & $0.05 \leq \mathrm{RMSEA}<0.08$ & $0 \leq \mathrm{RMSEA}<0.05$ \\
\hline HOELTER & 251 & - & - \\
\hline
\end{tabular}

were correlated [11]. Errors were considered as a hidden variable because they were not measured directly [12]. To determine the fitness of the structural equation model, the most famous indices including chi-square index, Goodness of Fit Index (GFI), the adjusted Goodness Of Fit Index (AGFI), etc. were used [13].

Four variables of physical activity, demographic information, contraceptive methods and fertility pattern were examined. Data were analyzed by Amous software V. 11.

\section{Results}

In this study, the most common method of contraception used was the natural method (73.2\%). Income level had a positive and significant relationship with the variables of using pills, condoms, IUDs, natural prevention methods, walking, and other physical activities $(\mathrm{P}<0.05)$. Secondary infertility had no significant relationship with the variables of condom use, 3-month injection, IUD and other physical activities $(\mathrm{P}<0.05)$.

The results of fitting the structural equation model showed that the indicators related to the proposed model of $X^{2} / \mathrm{df}$ was significant $(\mathrm{P}<0.001)$, which expressed an acceptable fitness. The values of GFI, AGFI and $\mathrm{CFI}^{1}$ were close to the value of 1 , which indicated the optimal fitness of the model. The value of RMSEA ${ }^{2}$ in this table was 0.06 , which according to the relevant studies, this value has always been a desirable model if it is less than 0.1 (Table 1).

Figure 2 is the approved model of the structural equation model of the four variables: the fertility pattern, contracep-

1. Comparative Fit Index

2. Root Mean Square Error of Approximation tive methods, physical activity, and demographic information. Demographic factors including occupation, income level and place of residence affected fertility pattern. Contraceptive methods were also effective on fertility pattern. Among the contraceptive subscales, condom use subscale was the most effective. Physical activity had the greatest effect on fertility pattern among other hidden variables.

\section{Discussion}

Structural equation method was used to identify the factors affecting the pregnancy pattern [14]. Most of the women in this study were housewives and their husbands had low incomes, which is consistent with other studies [15, 16]. Income level had a positive and significant relationship with primary and secondary infertility that is consistent with the results of a survey conducted by the Statistics Center of Iran [17]. Other studies showed that low-income people are less likely to have children [18-20], which is not consistent with the present study.

In the present study, $10.4 \%$ of the subjects had primary infertility and $14.1 \%$ had secondary infertility. In Khademi et al.'s study, $93.2 \%$ of the subjects had primary infertility and $6.8 \%$ had secondary infertility [21]. In other studies, the percentage of people with primary and secondary infertility was less than the amount obtained in this study [22, 23]. The duration of treatment in the present study had a significant effect on the fertility pattern [24]. Mora et al. showed that the duration of treatment had no significant relationship with fertility [25]. The reason for this discrepancy may be due to differences in the choice of contraceptive method. In the present study, there was a significant relationship between treatment methods such as intracytoplasmic sperm injection, in vitro fertilization and other methods [26]. The 
use of contraceptive methods was not mentioned as a cause of infertility [27].

In the present study, the natural method, condom use and pill use, respectively, were the most common contraceptive methods, which is consistent with other studies [31-33]. The reason for using different methods of contraception can be due to the geographical residence of people. Women who were overweight and sedentary experienced infertility $[32,33]$. Therefore, by recognizing the factors affecting the fertility pattern and increasing the awareness of young couples, it is possible to help improve the fertility pattern.

\section{Ethical Considerations}

\section{Compliance with ethical guidelines}

This article was approved by the Research Council of Arak University of Medical Sciences (No. 1727 and Ethics Committee ID: IR.ARAKMU.REC.1396.4).

\section{Funding}

The present paper was extracted from the MSc. thesis of the first author, Department of Biostatistics, School of Medicine, Arak University of Medical Sciences, Arak.

\section{Authors' contributions}

All authors were equally contributed in preparing this article.

\section{Conflicts of interest}

The authors declared no conflict of interest.

\section{Acknowledgements}

We appreciate the cooperation of the Vice Chancellor for Research and Technology of Arak University of Medical Sciences. 
This Page Intentionally Left Blank 


\title{
بررسى عوامل مؤثر بر الكَوى بارورى شهر اراك در سال جوسا با مدل معادلات ساختارى
}

\author{
فاطمه اميرى' ه. "رسول نجفى' ه \\ 1. كروه آمار زيستى، دانشكده يزشكى، دانشكاه علوميزشكى اراى، اراك، ايران.
}

\begin{abstract}
سيكين

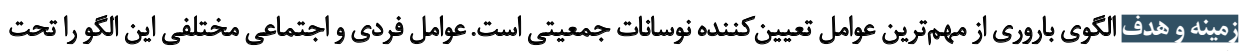

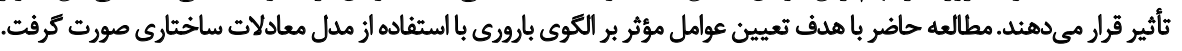

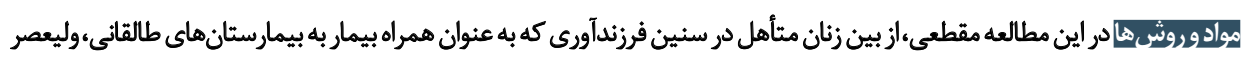

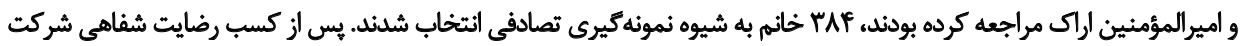

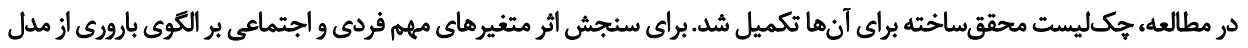

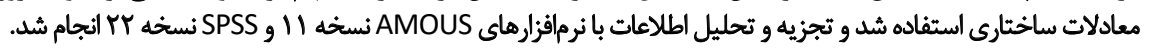

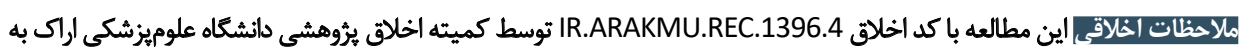
ثبت رسيد.

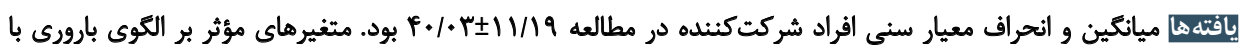

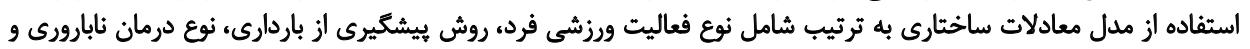

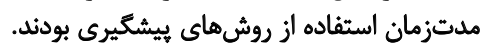

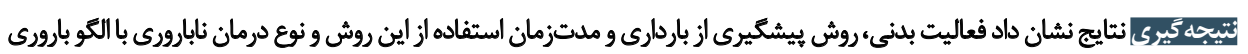

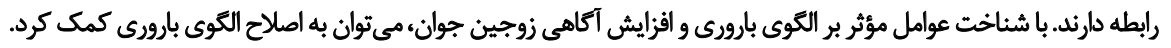

اطلاعات مقاله:

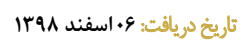

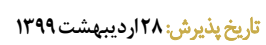

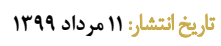

\section{كليدواروهها:

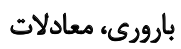

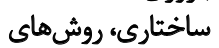 بيشكيرى از باردارى}

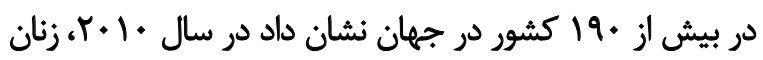

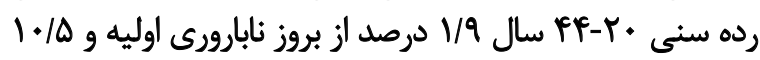

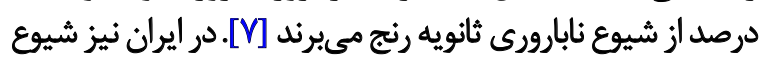

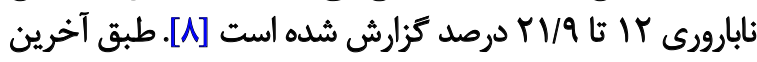

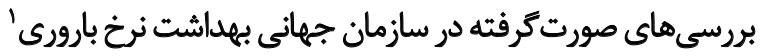

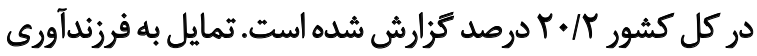

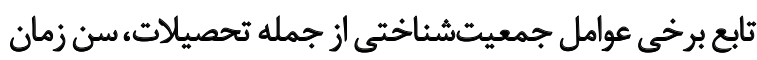

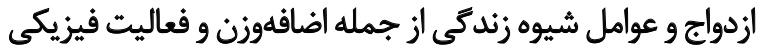

است [9].

يكى از راههاى تعيين عوامل مؤثر بر الثوى بارورى استفاده از مازي

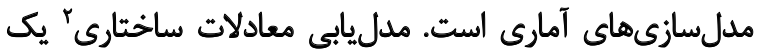

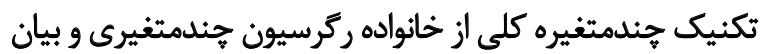

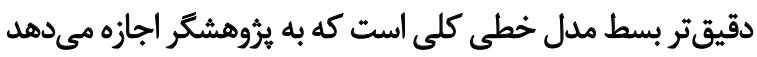

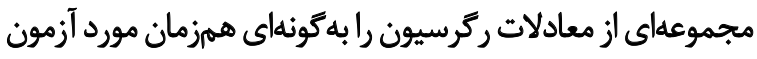

1. Fertility rate

2. Structural Equation Modeling (SEM)

مقدمه

الكوى بارورى مهمثرين بديده تعيين كنتده نوسانات جمعيت

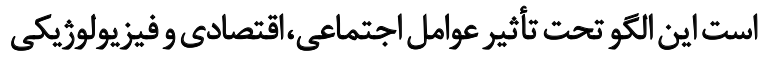

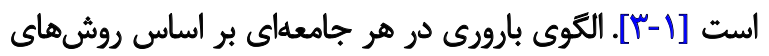

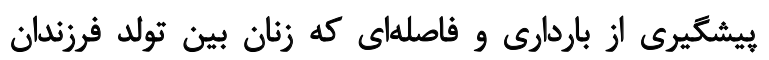

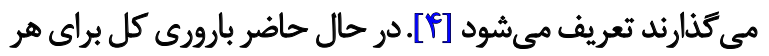

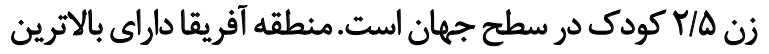

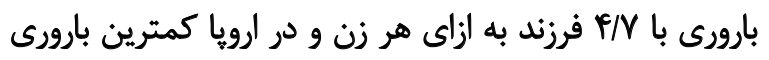

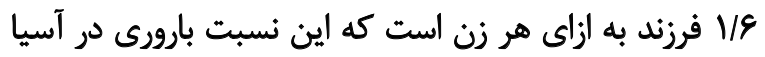

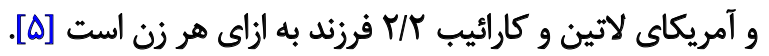

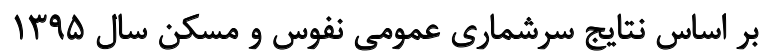

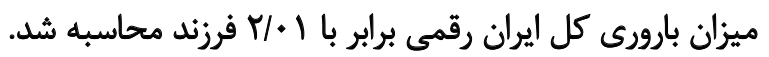

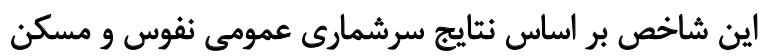

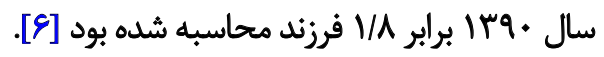
نابارورى يك مسئله محورى براى زوجهاى در سنين باني بارورى سري

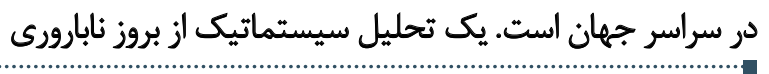




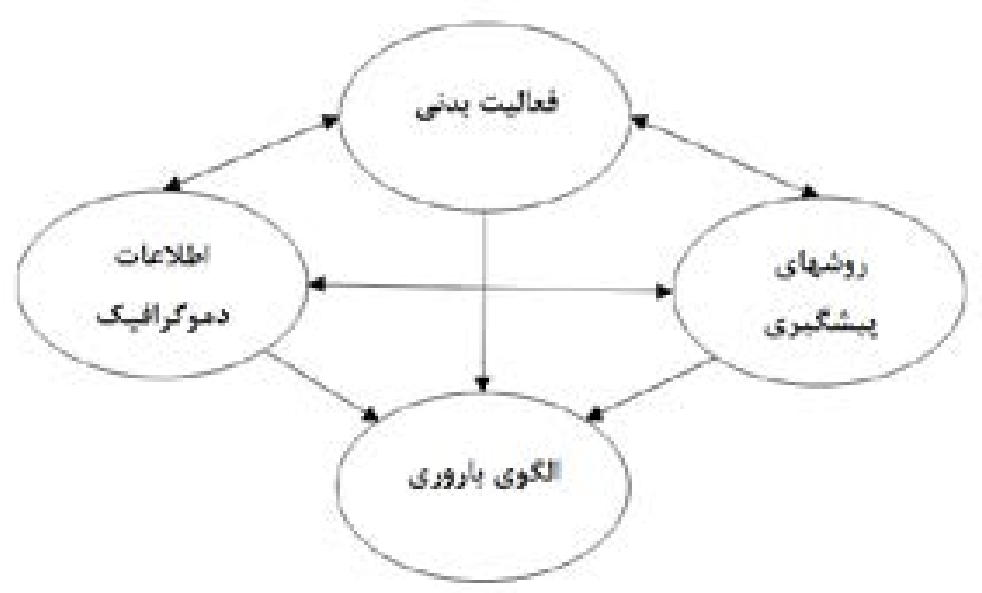

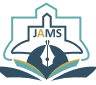

ديغر متغيرها قابل محاسبه است [11]]. مدلهاى ساختارى

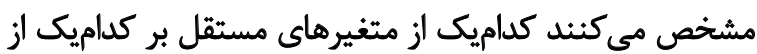

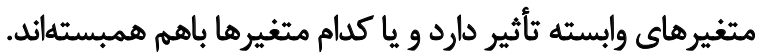

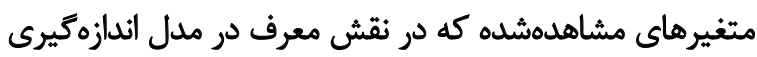

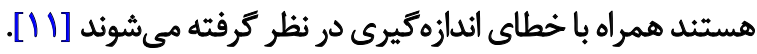

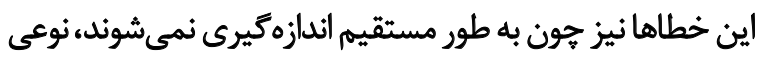

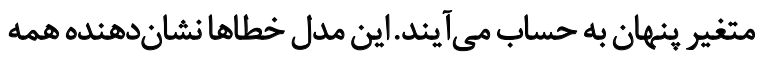

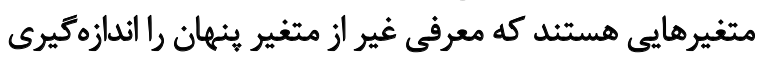

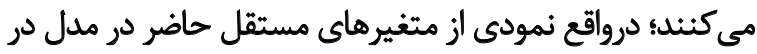

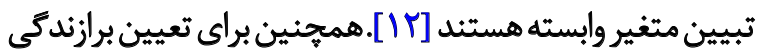

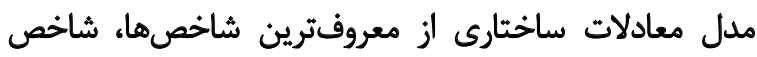

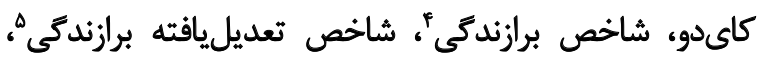

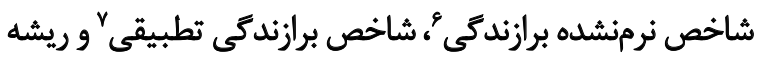

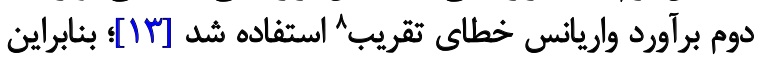

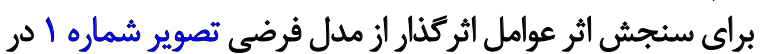
روابط متغيرها استفاده شد:

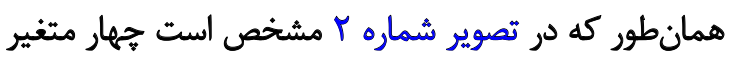

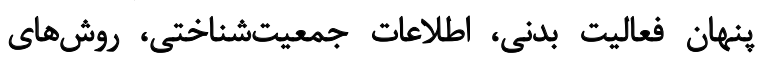

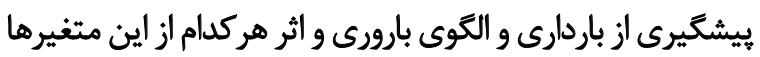

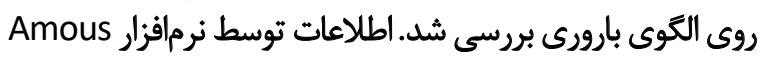

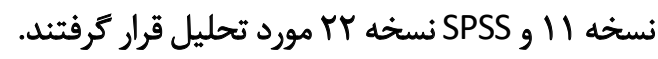

يافتهها

ميانتين و انحراف معيار سنى rAF خانم شركت كنتده در

\section{Goodness of Fit Index (GFI)}

5. Adjusted Goodness of Fit Index (AGFI)

6. Normed Fit Index (NFI)

7. Comparative Fit Index (CFI)

8. Root Mean Square Error of Approximation (RMSEA)
تصوير ا. مدل مفهومى فرضى از روابط بين متغيرها

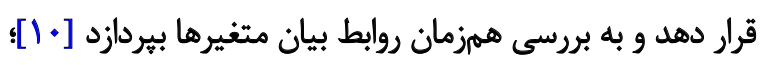

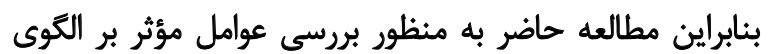

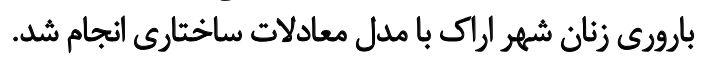

\section{مواد و روشّها}

مطالعه حاضر يك مطالعه مقطعى است كه جامعه آمارى آن

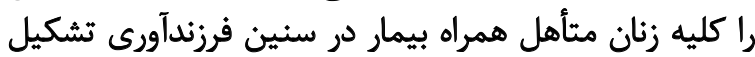

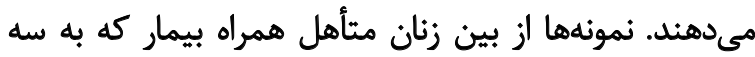

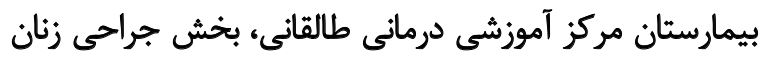

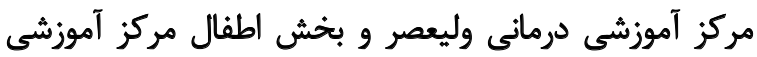

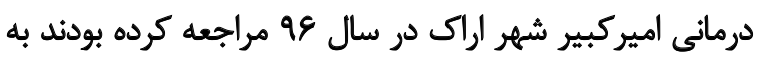
صورت تصادفى انتخاب شدند.

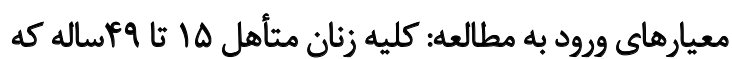

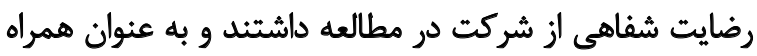

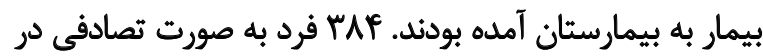

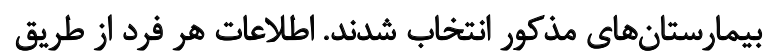

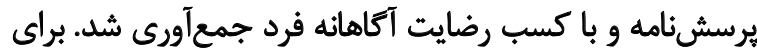

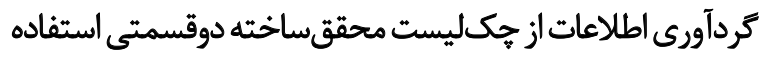

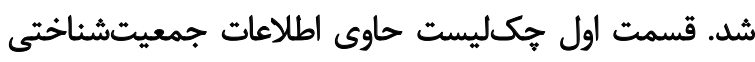
(سن، تحصيلات، محل سكونت، شغل و سطح درائ آمد) و وقسمت

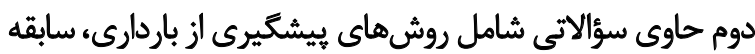

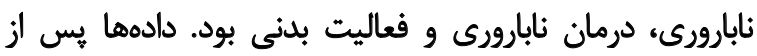

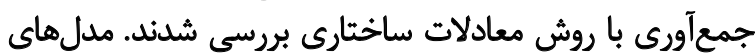

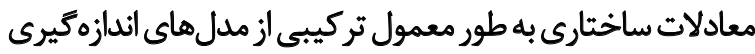

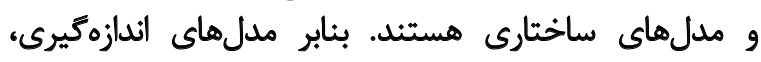

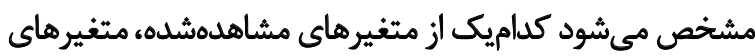

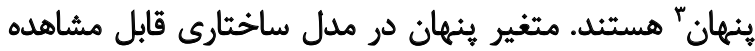
نيست و در برابر متغير آشكار قرار مى مئيرد. اين متغير توسط مابل مشامده

3. Latent Variable 


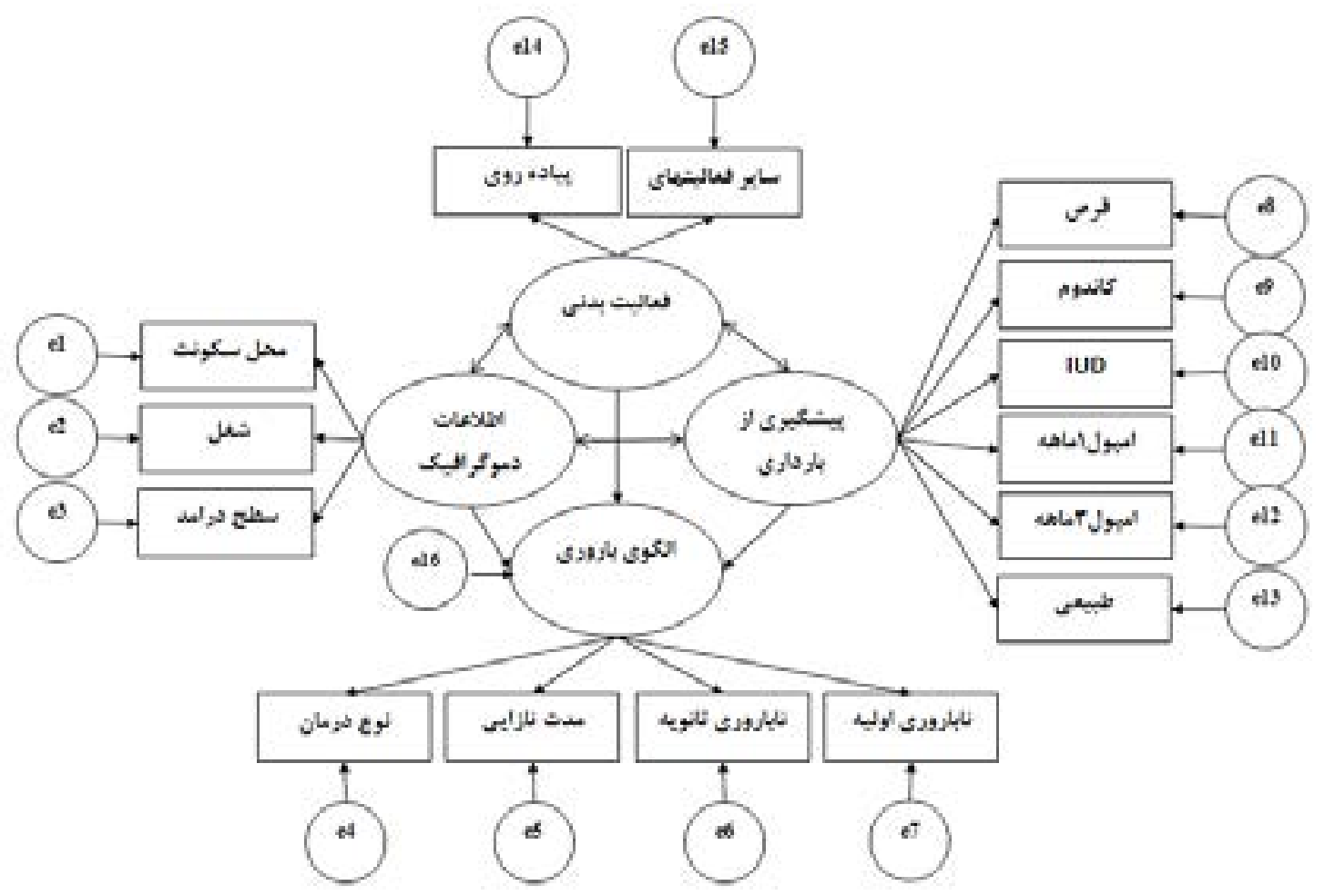

تصوير ז. الكوى بارورى، روشهاى بيشكيرى از بارورى، فعاليت بدنى و اطلاعات جمعيتشناختى (محل سكونت، شغل و سطح درآمد)

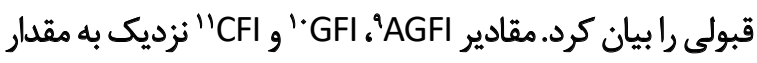

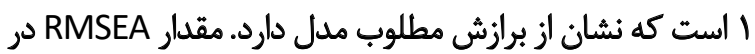

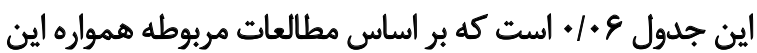

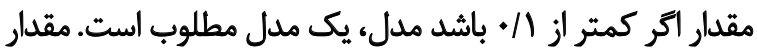
HOELTER حجم نمونه در اين مطالعه نيز مناسب است (جدول شماره سب). از آنجا كه در روش معادلات ساختارى ميتوان همزمان جند ميند

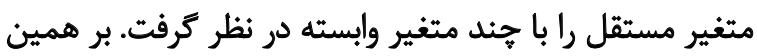

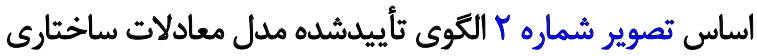

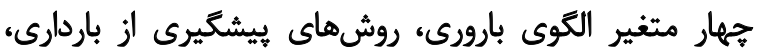

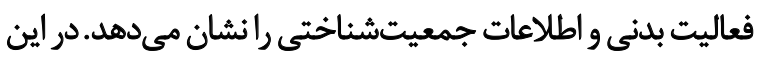
مدل تأييدشده تمام وزنهاي ركرسيوني معنادار هستيند. عوامل

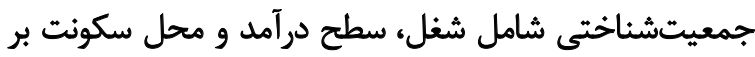

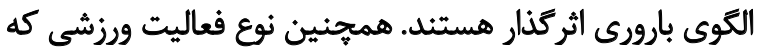

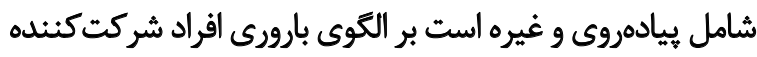

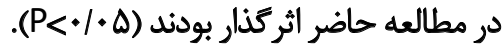

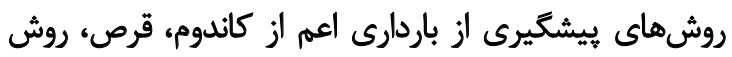

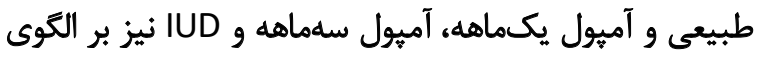

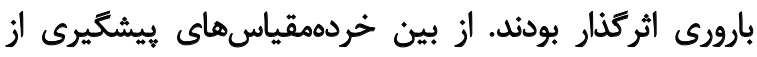

9. Adjusted Goodness of Fit Index

10. Goodness of Fit Index

11. Comparative Fit Index

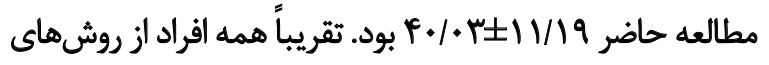
بيشيرى از باردارى استفاده كرده بودند، اما انتخاب و و درصد

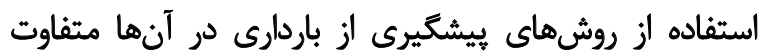

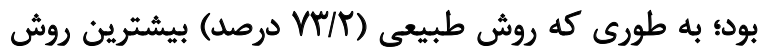

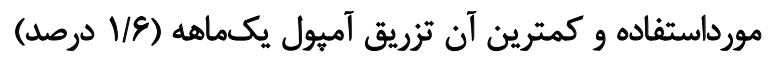

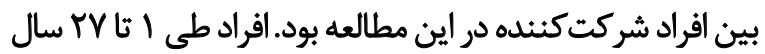

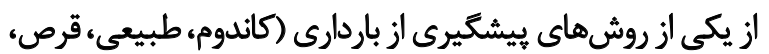

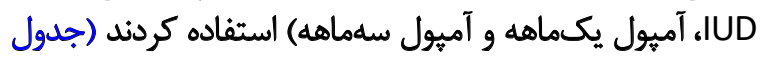

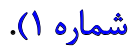

در جدول شماره Y، همبستكى هاي ميان متغيرها با استفاده

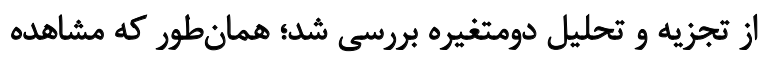

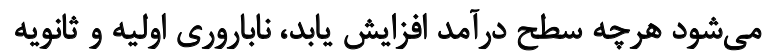

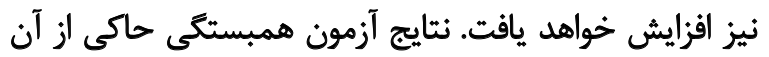

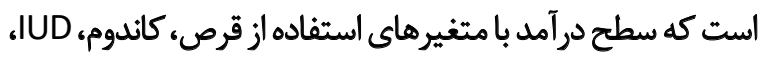

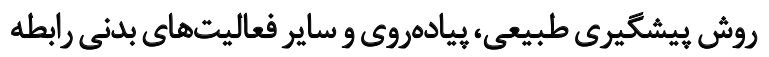

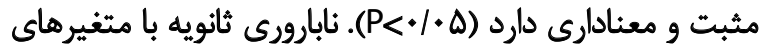

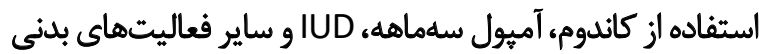

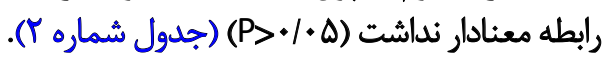

نتايج حاصل از برازش مدل معادلات ساختارى در جدول شماره

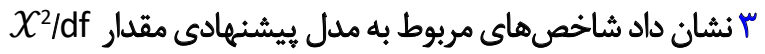

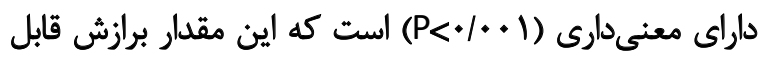


جدول ا. اطلاعات جمعيتشناختى و روش هاى بيشكيرى از باردارى

\begin{tabular}{|c|c|c|}
\hline تعداد (دوصد) & $\operatorname{los}_{g} 5$ & مثغيرها \\
\hline $\operatorname{lof}(f+/ 1)$ & ها تا عب سال & \multirow{3}{*}{ سن } \\
\hline $\operatorname{lg\lambda }(\mathrm{FT} / \Lambda)$ & عr تا VT سال & \\
\hline$E(\mid \& /)$ & & \\
\hline$M(M V / \Delta)$ & ل - ليىسواد & \multirow{3}{*}{ تحصيلات } \\
\hline $\operatorname{Mr}(\Delta Q / 1)$ & زير دييلم & \\
\hline$r e(q / \varphi)$ & مانشكاهي & \\
\hline $\operatorname{meg}(9 . / 1)$ & كمتر از •..... ه تومان & \multirow{3}{*}{ سطع درامد } \\
\hline$M(\Delta / M)$ & •.............. اتومان & \\
\hline $1 \notin(f / T)$ & بيشتر از +...... تومان & \\
\hline$r_{+}+(V N)$ & شهر & \multirow{2}{*}{ مجل سكونت } \\
\hline$A F(T / M)$ & روستا & \\
\hline $\operatorname{rIA}(\Delta F / \wedge)$ & ييادهروى & \multirow[b]{2}{*}{ فعاليت بلنيى } \\
\hline $\operatorname{leg}(R T / T)$ & تساير & \\
\hline IFq (TNA) & 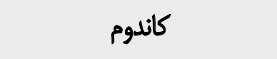 & \multirow{6}{*}{ روش هاى ييشكيرى أز باردارى } \\
\hline rA $(N / T)$ & IUD & \\
\hline 111 (TNQ) & قرص & \\
\hline$\varphi(1 / \Delta)$ & آميول يكماهد & \\
\hline $1 \cdot(r / 8)$ & آميول سلهماهد & \\
\hline$r A)(N / 1)$ & طييعى & \\
\hline$r \Delta q(9 r / \Delta)$ & خانهدار & \multirow{2}{*}{ شغل } \\
\hline$\|(T / 9)$ & آزاد & \\
\hline
\end{tabular}

شد كه يّ از برديى با استفاده از روش معادلات ساختارى

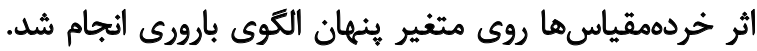

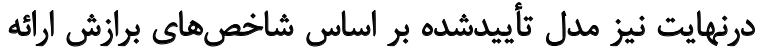

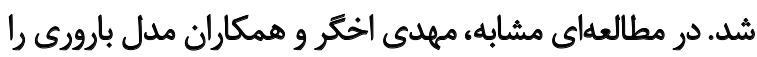

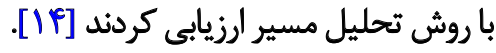

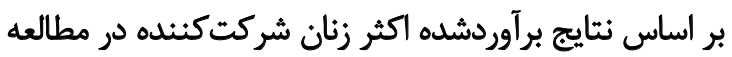

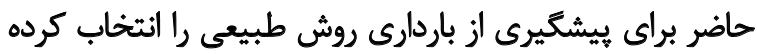

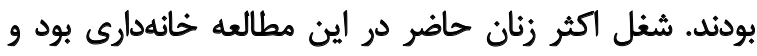

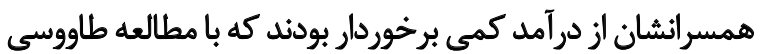

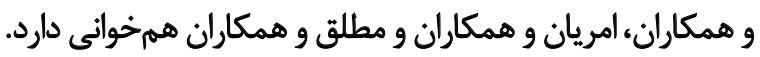

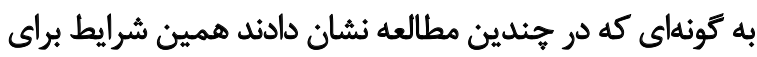

باردارى خردهمقياس استفاده از كاندوم بيشترين تأثير را دارد.

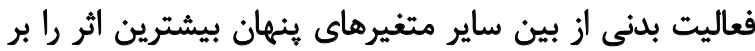

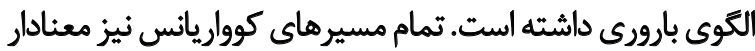

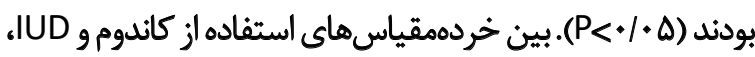

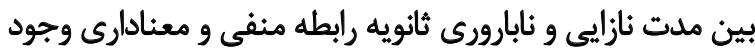

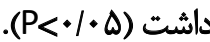

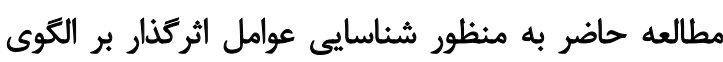

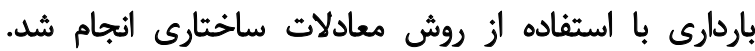

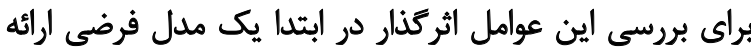


جدول ץ : همبستكى بين خردهمياس هاى اطلاعات جمعيتشناختى، فعاليت بدنى، روش هاى بيشكيرى از باردارى و الكّوى بارورى

\begin{tabular}{|c|c|c|c|c|c|c|c|c|c|c|c|c|}
\hline ir & 11 & 1. & 9 & $\Lambda$ & $r$ & 7 & $\bullet$ & $\varepsilon$ & $r$ & $r$ & 1 & هثغير \\
\hline & & & & & & & & & & & 1 & سطح درآمد \\
\hline & & & & & & & & & & 1 &.$/ 10^{* *}$ & قرص \\
\hline & & & & & & & & & 1 & $-* / \cdot r^{*}$ &.$- / / r^{* * *}$ & كائلدوم \\
\hline & & & & & & & & 1 & -.1 .1 & .1 .8 & $-.1+1$ & أميول (ماهنه \\
\hline & & & & & & & 1 & $-.1+r$ &.- .1 .8 & $-.1 . r$ & -.1 .8 & اميول "rماهيه \\
\hline & & & & & & 1 & .1 .4 & $.10 f$ &.$/ 1 r^{*}$ & -.1 .9 & $-* / N r^{*}$ & IUD \\
\hline & & & & & 1 & $-+1+1$ &.$/ 1 r^{*}$ & -.1 .8 &.$- / M$ & $-\cdot / N F^{*}$ & $-* / M I^{*}$ & طبيعى \\
\hline & & & & 1 & $-+/+4$ & 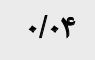 & $+/+V$ & $.1+1^{*}$ & $+/ 1 r^{*}$ & $+1+1$ &.$/ 1 r^{*}$ & يياده روى \\
\hline & & & 1 &.$- / 94 *$ & .1 .9 &.$- / 4$ & $+1+9$ & $+ハ^{*}$ & $-+/ 1 \Delta$ &.$/ 1 \%$ &.$/ 1 \Delta^{* *}$ & ساير \\
\hline & & 1 &.$- / 1$ & $+/+r^{*}$ & $-+1 .+{ }^{\circ}$ & $.1 .9^{*}$ & -.1 .8 & $-+1+F$ &.$- / .+*$ & $-+/ 18^{*}$ & $. / 1)^{*}$ & نابارورى اوليه \\
\hline & 1 & $-* / 11^{* *}$ & $.1 \cdot r$ & $-\cdot 1 \cdot r^{*}$ & $-.1 \cdot e^{p *}$ & $.1 \% \Delta^{*}$ & $.1 \cdot r^{*}$ & $-.1 \cdot \Delta^{*}$ & 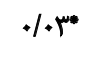 & .1 .9 &.$/ M$ & ثابارورى ثانويه \\
\hline 1 &.$- / 1 A^{\circ}$ &.$- / 4 T^{*}$ &.$/ 4$ &.- .1 .1 &.$/ 1 r^{*}$ & $.1 \cdot r$ & -.1 .4 & $1 \cdot v^{*}$ & .1 .0 &.$/ 1 \theta^{* *}$ & $1 . \Delta^{*}$ & مدت نازايع \\
\hline $.1 \cdot r$ & $.1+r$ &.$/ 10^{*}$ & $-.1 \cdot f$ & $.1 . f$ & $.1 \cdot r$ & $.1 \cdot 1$ & .1 .1 &.$- \cdot 1 \cdot 1$ & -.1 .8 & $.1 \cdot 1$ & $-* / \cdot e^{* *}$ & نوع درمان نازايى \\
\hline
\end{tabular}

جدول ". شاخصهاى برازش مربوط به مدل ييشنهادى

\begin{tabular}{|c|c|c|c|}
\hline دامنه ايدةآل & دامنه مورد قبول & مدل تدوين شُده & شاخص برازش \\
\hline $2 \mathrm{df} \leq / 2 x$ & $5 \mathrm{df} \leq / 2 X$ & $r / T r$ & $d f / 2 x$ \\
\hline $1>\leq G F \mid 95 / 0$ & $95 / 0>\leq G F \mid 9 / 0$ & .19 & GFI \\
\hline $1>\leq$ CFI 95/0 & $95 / 0>\leq$ CFI 9/0 &.$/ 9 v$ & CFI \\
\hline $1>\leq$ PCFI $95 / 0$ & $95 / 0>\leq$ PCFI $9 / 0$ & +19 & PCFI \\
\hline $1>$ SAGFI 95/0 & $95 / 0>\leq$ AGFI $9 / 0$ &.$/ 9 \Delta$ & AGFI \\
\hline 05/0 > SRMSEA 0 & $08 / 0>\leq$ RMSEA 05/0 & .1 .9 & RMSEA \\
\hline - & - & TAI & HOELTER \\
\hline
\end{tabular}

كونهاى كه افراد داراى وضع اقتصادى بالا متقاضى فرزند كمترى

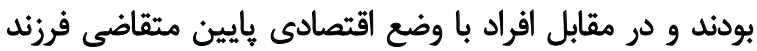

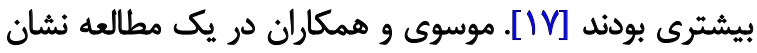

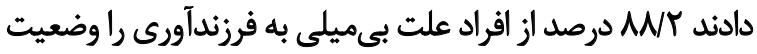

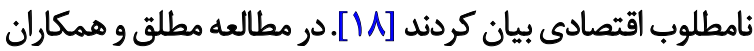

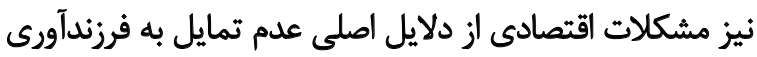

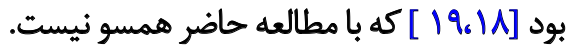

بررسى الكَوى بارورى برقرار است [ه| 18 ] ]. در مطالعه حاضر سطح درآمد با نابارورى اوليه و ثانويه داراى

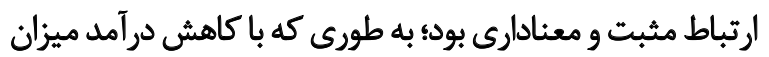

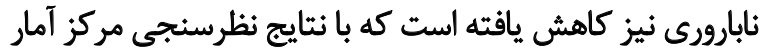

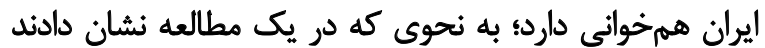

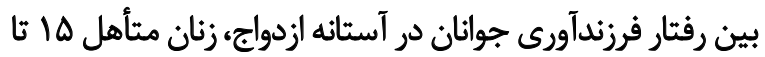

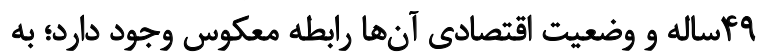




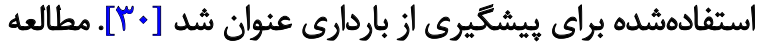

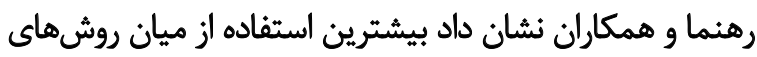

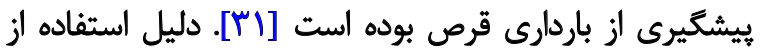

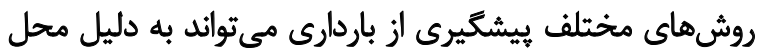

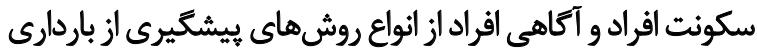

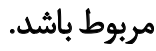

فعاليت بدنى روى بارورى خانمها تأثير داشت؛ بنابت بابراين

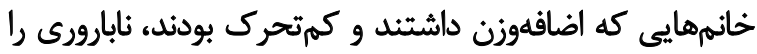

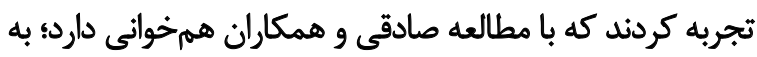

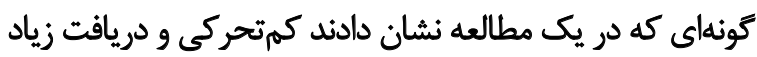

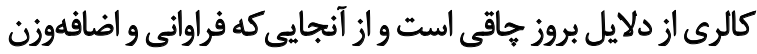

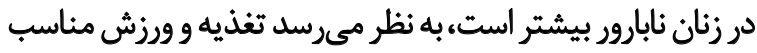

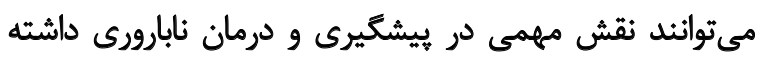

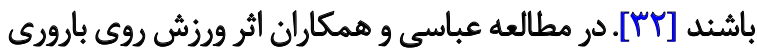

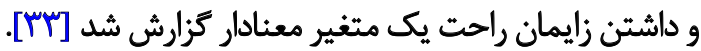

$$
\text { نتيجليكيرى }
$$

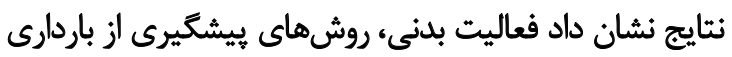

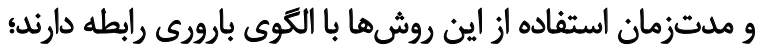

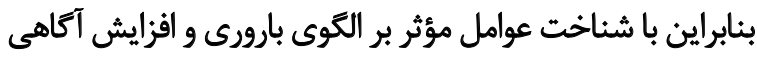

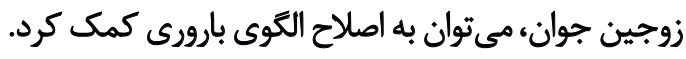

مالاحظات اخلاقى

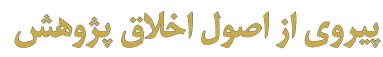

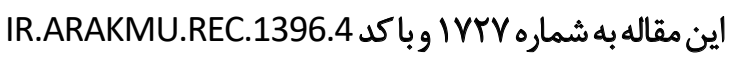
در كميته اخلاق دانشًاه علوم بزشكى اراك بار به تصويب رسيده

$$
\text { مانم }
$$

اين مقاله مستخرج از پايان نامه كارشناسى ارشد نويسنده اول

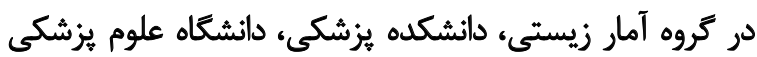

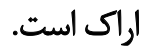

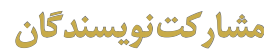

تمامى نويسندكان مذكور در نكارش اين مقاله به يك اندازه

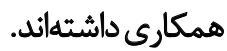

ثعارض مثأقع نويسندكان مقاله هيج گونه تعارضى در منافع اعلام نكردند.
شغل بر الكوى بارورى اثركذار بود كه با مطالعه ميرزايى و

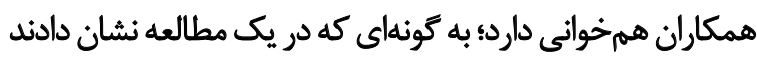

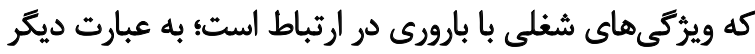

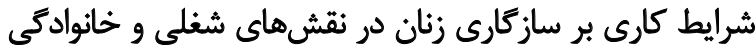

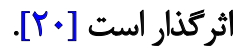

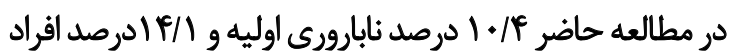

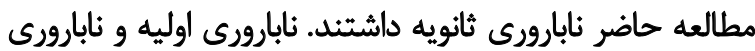

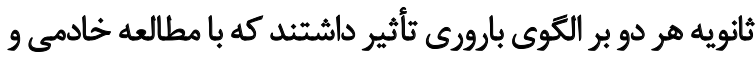

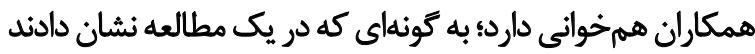

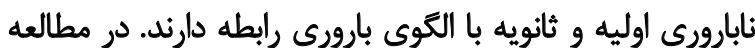

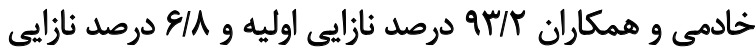

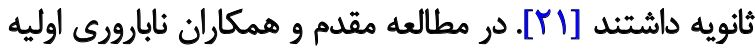

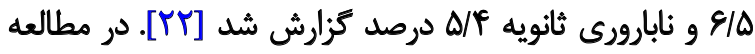

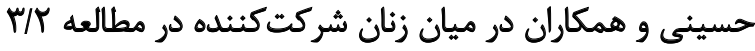

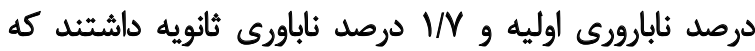

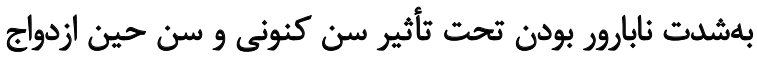

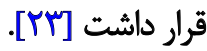

مدتزمان درمان در مطالعه حاضر روى الكوى بارورى اثر

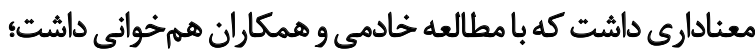

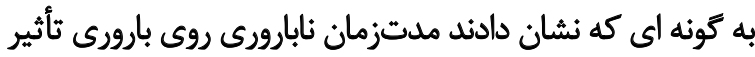

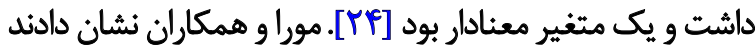

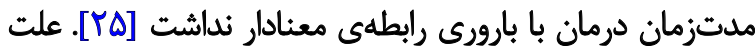

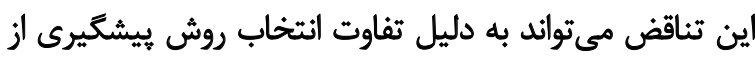

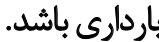

در مطالعه حاضر از بين روشهاي درمان نابارورى روش تزريق

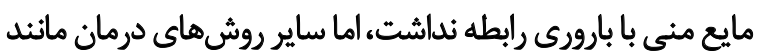

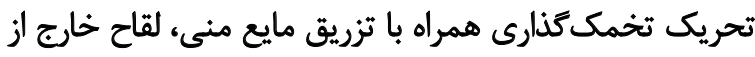

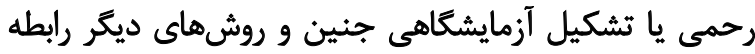

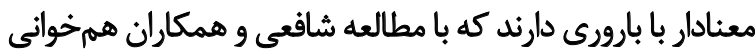

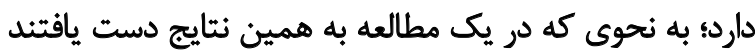

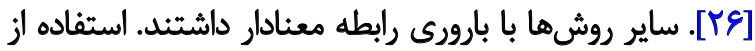

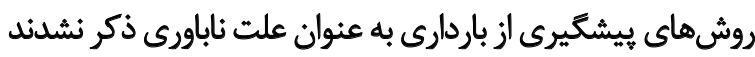

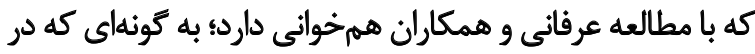

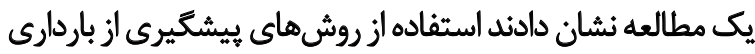

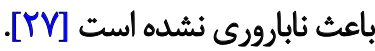

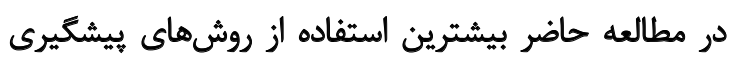

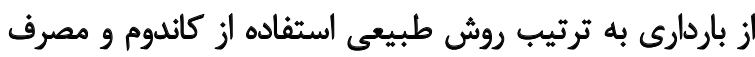

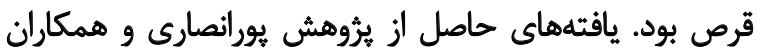

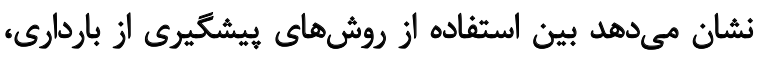

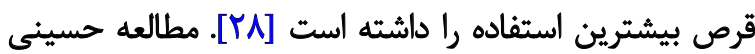

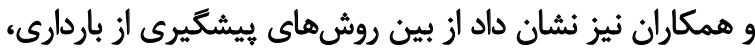
IUD

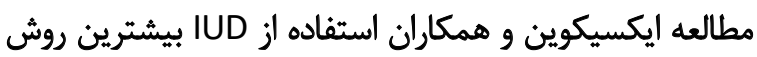




$$
\text { G) }
$$

بدينوسيله از همكارى معاونت محترم تحقيقات و فناورى دانشكاه علوميزُشكى اراك كمال تشكر و قدردانى را داريم. 


\section{References}

[1] Adhikari R. Demographic, socio-economic, and cultural factors affecting fertility differentials in Nepal. BMC Pregnancy Childbirth. 2010; 10(1):19. [DOI:10.1186/1471-2393-10-19] [PMID] [PMCID]

[2] Farrokh Eslamlou HR, Mogadam Tabrizi F, Moeini SR, Vahabzadeh Z. [Pre-marriage couplesfertility attitude following recent childbearing persuasive policies in Iran (Persian)]. J Urmia Nurs Midwifery Fac. 2013; 11(10):836-46. http://unmf.umsu.ac.ir/article-1-1649-fa.html

[3] Amiri F, Rafiei M, Najafi R. [Study of fertility and use of pregnancy contraceptive methods in women referring to remedial centers in Arak city in 2017 (Persian)]. J Arak Univ Med Sci. 2019; 22(3):1-0. http://jams.arakmu.ac.ir/article-1-5947-en.html

[4] Yadava RC, Kumar A, Pratap M. Estimation of parity progression ratios from open and closed birth interval data. J Data Sci. 2013; 11(3):607-21.

[5] Bagirova A, Shubat AB. Factors affecting reproductive activity of the population of russian regions. Reprodukce lidského kapitálu vzájemné vazby a souvislosti. 2014; 27-35.

[6] Fertility and infertility. Statistical Centre of Iran. [Internet]. 2019 [Updated 2019 Sept 08]. Available from: https://www.amar.org.ir/ english/Statistics-by-Topic/Population

[7] Cong J, Li P, Zheng L, Tan J. Prevalence and risk factors of infertility at a rural site of northern China. PloS One. 2016; 11(5):e0155563. [DOI:10.1371/journal.pone.0155563] [PMID] [PMCID]

[8] Fooladi E, Danesh MM, Kashfi F, Khani S, Mohammadpor RA. [Study of infertile husbands' and wives' point of view to infertility and marital adjustment in patients reffering to Royan infertility center of Tehran in 2005 (Persian)]. J Mazandaran Univ Med Sci. 2006; 16(55):131-7. http://jmums.mazums.ac.ir/article-1-162-fa.pdf

[9] World Health Organization. Global Health Observatory country views. [Internet]. 2002. [Updated 2020 Agu 07]. Available from: http://apps.who.int/gho/data/node.country.country-IRN.

[10] Alavi M. [Structural Equation Modeling (SEM) in health sciences education researches: An overview of the method and its application (Persian)]. Iran J Med Educ. 2013; 13(6):519-30. http://ijme.mui. ac.ir/article-1-2228-fa.htm

[11] Gunnell DJ, Ewings P. Infertility prevalence, needs assessment and purchasing. J Public Health. 1994; 16(1):29-35. [DOI:10.1093/oxfordjournals.pubmed.a042931] [PMID]

[12] Fskhodi AA, Hadinejad B. [Organizational effectiveness assessment with structural equation modeling approach (Persian)]. Res

Manage Iran. 2012; 2012(3):1-20. http://ensani.ir/file/download/ article/20121210084940-9566-31.pdf

[13] Hooman HA. Structural equation modeling with LISREL application. Tehran: SAMT; 2010. p. 340.

[14] Mahdi Akhgar M, Soltanian A, Mahjub H, Karami M. [Evaluation of fertility model by using path analysis in Hamadan city in 2014 (Per-

sian)]. Avicenna J Clin Med. 2015; 22(2):122-8. http://sjh.umsha.ac.ir/ article-1-42-en.pdf

[15] Tavousi M, Motlagh Me, Eslami M, Haerimehrizi A, Hashemi A, Montazeri A. [Fertility desire among Iranians living in Tehran: Rea-

sons for desire and disinterest (Persian)]. Payesh. 2016; 15(6):663-8. https://payeshjournal.ir/article-1-141-fa.pdf
[16] Amerian M, Kariman N, Janati P, Salmani F. [The role of individual factors in decision making for the first childbearing (Persian)]. Payesh.

2016; 15(2):143-51. https://payeshjournal.ir/article-1-182-fa.pdf

[17] Statistical Center of Iran. Fertility desire in adolescents. [Internet] 2014 [Updated 2020 Sep 08]. Availble from: https://www.amar.org.ir/ english/Statistics-by-Topic/Education-and-Research

[18] Mousavi SF. The Role of interpersonal identity statuses in predicting the attitudes of married people toward love. IInt J Psychol. 2014; 8(2):74-88. http://www.ijpb.ir/article_55578_52264d9bd6cd16a67fd9 f53eff526109.pdf

[19] Keshavarz H, Haghighatian M, Tavasoli Dinani Kh. [A study on the factors influencing the space between marriage and having children (Case study: Married women of 20-49 in Isfahan) (Persian)] J Appl Soci Univ Isfahan. 2013; 24(2):111-26. http://jas.ui.ac.ir/article_18305.html

[20] Mirzaei M, Shams Ghahfarrokhi M. [The effect of women's employment characteristics on fertility (Persian)]. Hum Reprod. 2014 9(17):113-39. http://www.jpaiassoc.ir/article_20071_289448803cfe79 8f086df8666ae4a3a7.pdf

[21] Khademi A, Alleyassin A, Agha-Hosseini M, Ahmadi Abhari A Esfhandi $H$, Fakhimi Derakhshan K. [Depression and infertility: Evaluation of 681 infertile women using Beck depression inventory (pERSIAN)]. J Hayat. 2004; 10(2):13-21. http://hayat.tums.ac.ir/article1-264-fa.html

[22] Sadegh Moghadam L, Moslem AR, Gharche M, Chamanzari H. [Study of women infertility of Gonabad (Persian)]. Horiz Med Sci. 2008; 13(4):82-5. http://hms.gmu.ac.ir/article- - -r. . -fa.html

[23] Hosseini J, Emadedin M, Mokhtarpour H, Sorani M. Prevalence of primary and secondary infertility in four selected provinces in Iran, 2010-2011. Iran J Obstet Gynecol Infertil. 2012; 15(29):1-7. http:// eprints.mums.ac.ir/id/eprint/5542

[24] Khademi A, Alleyassin A, Aghahosseini M, Ramezanzadeh F, Abhar AA. Pretreatment Beck Depression Inventory score is an important predictor for post-treatment score in infertile patients: a before-after study. BMC Psychiatry. 2005; 5(1):25. [DOI:10.1186/1471-244X-5-25] [PMID] [PMCID]

[25] Moura-Ramos M, Gameiro S, Canavarro M, Soares I. Assessing infertility stress: Re-examining the factor structure of the Fertility Problem Inventory. Hum Reprod. 2011; 27(2):496-505. [DOI:10.1093/ humrep/der388] [PMID]

[26] Shafaie FS, Mirghafourvand M, Rahimi M. Perceived stress and its social-individual predicors among infertile couples referring to infertility center of Alzahra hospital in Tabriz in 2013. Int J Womens Health Reprod Sci. 2014; 2(5):291-6. [DOI:10.15296/ijwhr.2014.47]

[27] Erfani A. Curbing publicly-funded family planning services in Iran: Who is affected? : J Fam Plann Reprod Health Care. 2017; 43(1):3743. [DOI:10.1136/jfprhc-2015-101383] [PMID]

[28] Pouransary Z, Sheikh Z, Eshrati B, Kamali P. [Prevalence of contraceptive use and the importance of the husband's cooperation in married women in Iranshahr, 2005 (Persian)]. Iran J Epidemiol. 2008 3(3):55-60. https://irje.tums.ac.ir/article-1-162-en.pdf

[29] Hosseini N, Mazloomy S, Fallahzadeh H, Sharifabad M. [Study of continuance rate and related causes of discontinuance of pregnancy prevention methods among women in Yazd (Persian)]. SSU_J . 2008; 16(1):166. http://jssu.ssu.ac.ir/article-1-552-en.pdf 
[30] Xiaoqin C, Li X, Xiaoping P, Chuanrong Z, Shiyuan L. Survey of IUD replacing status in Sichuan, PR China. Contracept. 2003; 67(5):37983. [DOI:10.1016/S0010-7824(03)00003-9]

[31] Rahnama P, Hidarnia A, Montazeri A, Kazemnejad A. Salient beliefs relevant to oral contraceptives among withdrawal users for development of a Belief-Based Questionnaire: Applying the theory of planned behavior. J Qual Res Health Sci. 2012; 1(3):202-13. http://jgr. kmu.ac.ir/article-1-87-fa.html

[32] Sadeghi F, Miri N, Barikani A, Hossein Rashidi B, Ghasemi Nejad A, Hojaji E, et al. [Comparison of Food intake, physical activity and weight in infertile and healthy women aged 25-40 years (Persian)]. Iran J Obstet Gynecol Infertil. 2015; 18(173):32-40. http://ijogi.mums.ac.ir/article_ 6367_103b49ded984bee7ecf4430d3984a8c3.pdf

[33] Abbasi S, Moazami M, Bijeh N, Mirmajidi SR. [Investigation of the Relationship between Physical Activity Levels, Maternal Weight (before delivery) and Serum Cortisol Level (during labor) in Nulliparous women (Persian)]. Iran J Obstet Gynecol Infertil. 2015; 18(151):12-9. http://ijogi. mums.ac.ir/article_4625_6baf8da12cba5e93a4d428d32a42e280.pdf 